\title{
EL TRABAJO DE LAS EMOCIONES EN LOS CONTENIDOS DE EDUCACIÓN FÍSICA
}

\section{Carlos Vallés Ortega}

Diplomado en Magisterio de Educación Física, Licenciado en Ciencias de la Actividad

\section{Carlos.valles.ortega@hotmail.com}

\section{RESUMEN:}

Con el siguiente artículo queremos indagar en las múltiples opciones que nos proporciona la asignatura de Educación Física y sus contenidos para trabajar a través de ellas, las emociones, la inteligencia emocional y las inteligencia múltiples.

PALABRAS CLAVE: Educación Física y Emociones. 


\section{1.- INTRODUCCIÓN}

La inteligencia humana es mas rica y dinámica de lo que han hecho creer a la educación académica formal. (Ken Robinson 2010).

Esta afirmación la podríamos relacionar con una de las bases teóricas de las inteligencias múltiples de Garnet (1993); antecedente cultural e histórico donde en la época actual la predominancia de dos inteligencias, la lógica matemática y lingüística, relegan al rechazo de las demás, siendo incluso un obstáculo a nivel académico para muchos docentes o gobernantes intentando eclipsarlas con reducción de horas a favor de las mencionadas u olvidadas en el baúl de los recuerdo con una desaparición de las mismas.

Objetivos que perseguimos en esta investigación:

- Identificar las emociones presente en los contenidos de educación física

- Utilizar la inteligencia emocional para potenciar las emociones en los diferentes contenidos

- Demostrar la importancia de la inteligencia múltiple, corporal, kinestésica para el trabajo de las emociones

- Conocer autores que siguen estas líneas de investigación

Antes de meternos de lleno con el trabajo empecemos definiendo y distinguiendo tres concepto básicos en el desarrollo de este estudio como son las emociones, las inteligencias emocionales y las inteligencias múltiples.

\section{2.- LAS EMOCIONES}

Siguiendo a Rueda (2011), podemos considerar la emoción como la habilidad de las personas para atender y percibir los sentimientos de forma apropiada y precisa, la capacidad para asimilarlos y comprenderlos de manera adecuada, y la destreza para regular y modificar nuestro estado de ánimo o el de los demás.

Esta capacidad que tenemos los seres humanos para sentir determinadas emociones constituye un elemento biológico fundamental, ya que las emociones son indicadores que nos empuja a lo que nos produce bienestar o peligro. Estás sospechas son las que nos conduce a realizar la acción o el movimiento por lo tanto 
ser consciente de ellas será determinante en la toma de decisiones, o en nuestra vida cotidiana tanto a nivel personal como profesional.

El punto de referencia a nivel científico y máximo exponente por sus estudios es el investigador Luso-Américano Antonio R. Damasio. Estudiando las redes, sistemas, subsistemas, neurales en interrelación que soportan el funcionamiento de la memoria, del lenguaje, de las tomas de decisiones, pero, sobre todo, del conocimiento y de las emociones. Sin embargo la gran aportación de Damasio, es la neurologíapsicología de la emoción, explorando las estructuras y sistemas neuronales que permiten a los organismos de la sensación de si mismos y la producción consecuente de estados emocionales.

\section{3.- INTELIGENCIA EMOCIONAL}

Siguiendo la línea de Rueda (2011), podemos considerar la inteligencia emocional, ya no solo como la identificación y asimilación de los estados emocionales por parte del sujeto, entrando en juego el talento que tiene el sujeto para manejar esos estados emocionales a partir de los dos grandes modelos de desarrollo de la inteligencia emocional:

\section{1.- los modelos mixtos}

Hace referencia a la importancia de la personalidad en la regulación y el control de las emociones (Bar-On, 1997; Goleman, 1998).

\section{2.- el modelo de habilidad}

Se centra en el procesamiento emocional de la información, identificando la emoción que se da en esa situación, las competencias que podemos desarrollar para atender y percibir los sentimientos de forma apropiada y precisa, la capacidad para asimilarlos y manejarlos, la destreza para regular y modificar nuestro estado de ánimo o el de los demás (Mayer \& Salovey, 1997; Mayer, Caruso, \& Salovey, 1999; Bimbele, 2004).

\section{Dimensiones de la inteligencia emocional}

- Percepción, valoración, y expresión de la emoción. Identificación de nuestras emociones y de los que nos rodea, junto con la expresión de las mismas. 
- La emoción como facilitadora del pensamiento y de la toma de decisiones, determinando en muchas ocasiones como enfrentarnos al problema.

- Comprensión y análisis de las emociones como las relaciones entre ellas.

- Regulación de las emociones, estando abierto a los estados emocionales positivos como negativos.

\section{4.-INTELIGENCIAS MÚLTIPLES}

Modelo creado por Howar Gardner (1983) en el que las inteligencia no es vista como algo unitario, que agrupa a diferentes capacidades específicas con distinto nivel de generalidad, si no como un conjunto de inteligencias múltiples, definiéndola como la capacidad de resolver problemas o elaborar productos que sean valiosos en una 0 más culturas.

Distintas inteligencias:

- Inteligencia Musical, capacidad de percibir, discriminar, y transformar las formas musicales.

- Inteligencia Corporal-kinestésica, capacidad para usar todo el cuerpo en la expresión de ideas y sentimientos.

- Inteligencia Lingüística, don de emplear la palabra de forma correcta, ya sea oral o escrita.

- Inteligencia Lógico Matemática, el valor del razonamiento y resolución adecuada de los números.

- La Inteligencia Espacial, permite percibir imágenes externas e internas, recrearlas, transformarlas o modificarlas, recorrer el espacio o hacer que los objetos lo recorran y producir o decodificar información gráfica.

- La Inteligencia Interpersonal, es la capacidad de entender a los demás.

- La Inteligencia Intrapersonal, empieza desde el respecto a si mismo, a la vez de organizar su vida.

- Inteligencia Naturalista, utilización y relación con elementos del medio ambiente, objetos, animales o plantas.

Centrándonos en nuestra materia rompemos una lanza a favor de la Inteligencia Corporal Kinestésica, sin obviar las otras inteligencias pues a la hora de realizar actividad física siguiendo una de las bases teóricas de las inteligencias múltiples, operación central o conjunto de operaciones identificables, estamos desarrollando las otras. 
En la inteligencia musical, mediante la expresión corporal, inteligencia lingüística, con la comunicación del entrenador y los propios compañeros de equipo, la inteligencia lógica matemática, mediante las puntuaciones, con el trabajo táctico incidimos en la inteligencia espacial, la inteligencia interpersonal, en deportes de equipo, el desarrollo de la inteligencia naturalista, en las actividades físicas en el medio natural, y por último la inteligencia intrapersonal, con el desarrollo de las emociones que siente el sujeto a realizar una actividad.

\section{5.- PSICOLOGÍA FISIOLÓGICA}

Siguiendo Levav, (2005), podemos entender las estructuras anatómicas interconectadas por medio de redes neuronales, presente en el funcionamiento de las emociones, siendo estás las siguientes:

\section{Corteza paracingulada anterior}

Es el lugar donde se representa mentalmente las situaciones.

\section{Surco temporal anterior}

Es el responsable de la percepción de conductas planificadas y las señales sociales.

\section{Polos Temporales}

Lugar donde entra en juego la memoria, se detectan rostros y objetos conocidos.

\section{Amígdalas}

Ubicado dentro del lóbulo temporal, cercano al hipocampo, esta proximidad hace posible el recuerdo y sentimiento de las emociones. También tiene relación con la conducta y situaciones sociales, y entra en juego en las representaciones cognitivas y conductuales.

\section{Corteza prefrontal}

Multitud de acciones que se realiza en esta zona. Posibilita el cambio del foco de atención y la flexibilidad cognitiva, facilita la capacidad de adaptación a nuevas situaciones, contribuye al cambio de estrategia de acción, hace posible tomar en cuenta diferentes opciones en una situación nueva o vieja y facilita la medición de la conducta cooperativa. 
A nivel energético el desarrollo es bastante similar al físico, requiriendo fuente de energía para su funcionamiento.

Esta energía se obtiene de la depleción de glucosa en sangre, utilizando un $20 \%$ o $30 \%$ en reposo y entre el $60 \%$ a $80 \%$ para la comunicación entre neuronas y sus células de soporte, mientras que el resto de energía se emplea para exigencias momentáneas del cerebro con el medio. No tiene capacidad para almacenar sustrato energético por lo cual constantemente requiere suministro proveniente del torrente sanguíneo. La ingesta de glucosa reduce la fatiga mental y mejora las actividades cognitivas. Conde, (2011).

\section{6.-LOS ESTADOS EMOCIONALES}

El dominio de la táctica y técnica son acciones variables estable del rendimiento, que pueden ir mejorando en el tiempo, en cambio los estados emocionales de cualquier persona que realice alguna actividad motriz, son mas maleables o dependientes de las circunstancia que vive el individuo o grupo. Cuando nos enfrentamos a situaciones en donde el porcentaje de fracaso aventaja a terminar la actividad realizada con éxito, los pensamientos negativos predominan sobre los positivos, imposibilitando desarrollar de forma adecuada la destreza motriz en la que estemos centrada y a su vez imposibilitando su correcta realización y de esta forma disminuyendo el rendimiento. Este déficit de atención viene provocado por los pensamientos perturbadores, provocados por el fracaso, con respuesta emocionales negativas (ansiedad, temor, enfado, tristeza, etc...).

En el lado inverso, cuando la acción que se esta desarrollando tiene un alto porcentaje de terminarse con éxito, el sujeto piensa que tiene recursos necesarios para afrontarla y el fin de esta se suele desarrollar con eficacia Carrascosa (2003).

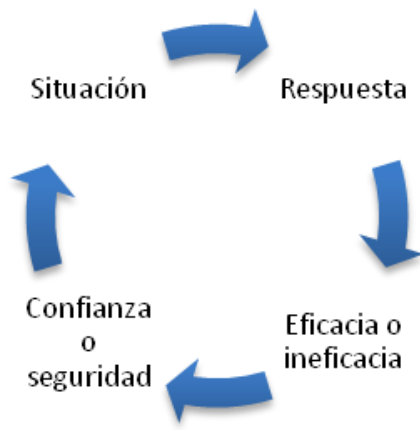

Figura 1 . Ciclo según la eficacia o ineficacia de la actividad. Carrascosa, (2003). 
Según lo expuesto con anterioridad las experiencia previas vividas por el sujeto son un elemento básico para una interpretación de pensamiento positivo o negativo de la acción que se va a desarrollar. Siguiendo a la RAE los pensamientos son "el conjunto de ideas propias de una persona o colectivo" activando respuesta emocionales concordantes. La respuesta a todo esto la encontramos en las bases neurofisiológicas del comportamiento, las cuales cuando se nos presenta una dificultad, activan los pensamientos negativos y a su vez estado emocionales destructivos impidiendo el rendimiento óptimo. Estos pensamientos distorsionan la realidad, generan problemas, alejando la posibilidad de satisfacer necesidades, y resolver con éxito la acción, reforzando de esta forma los estados emocionales negativos.

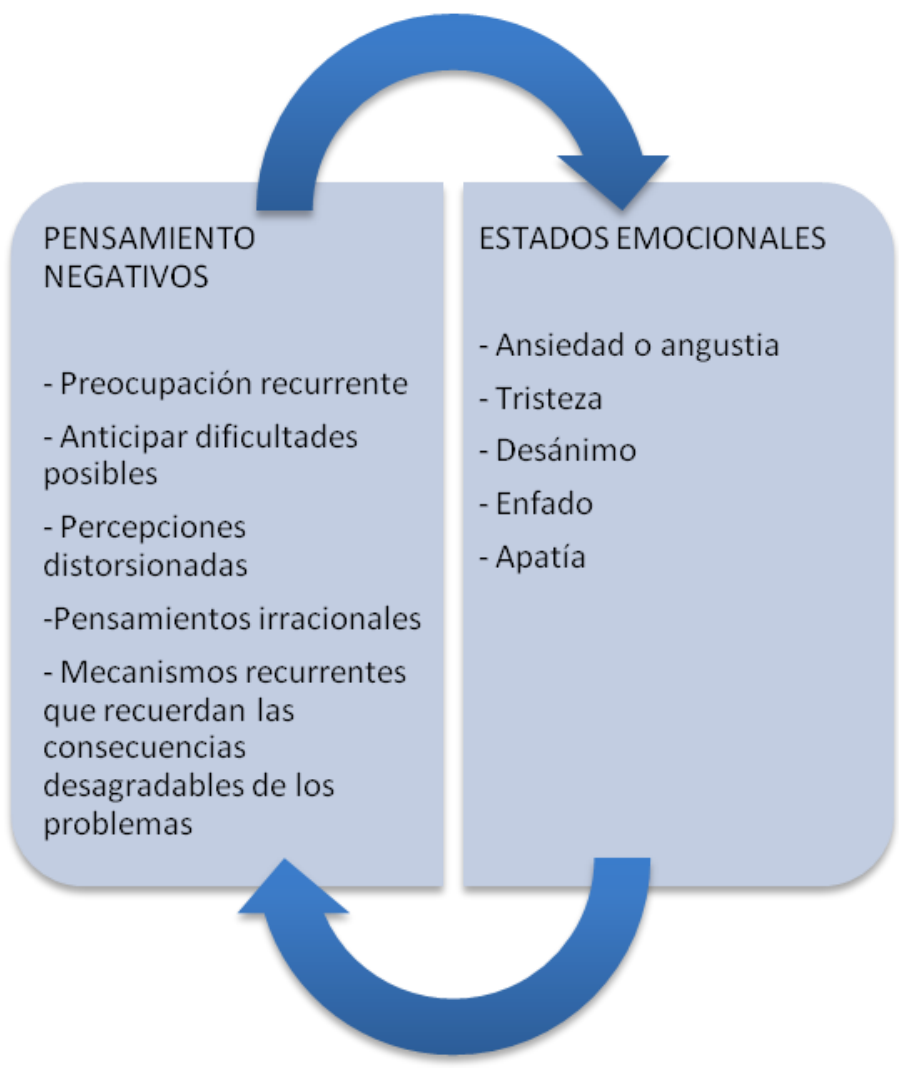

Figura 2 . Pensamos como sentimos. Carrascosa. J (2003).

Los pensamientos que recuerdan los recursos necesarios para hacer frente a la conflicto negativo son los concebidos por los estados emocionales positivos. Estos pensamientos reflejan la realidad objetiva, favoreciendo alcanzar los éxitos, y reforzando los estados emocionales positivos. 


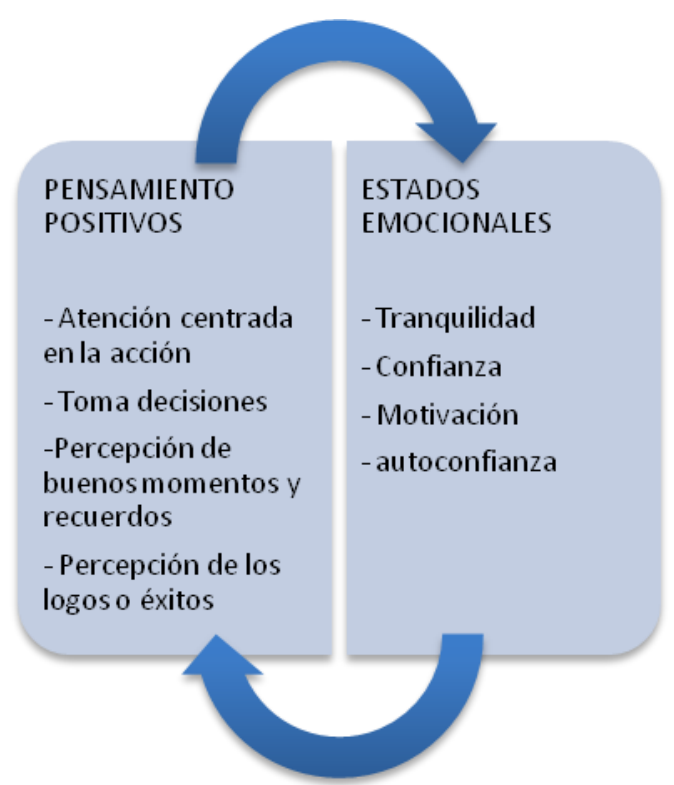

Figura 3. Pensamos como sentimos. Carrascosa, J (2003).

\section{7.-EDUCACIÓN FÍSICA Y EMOCIONES}

Después de mencionar los pensamientos que influyen en los estados emocionales de los sujetos en la práctica deportiva, intentaremos exponer los que se dan en los diferentes contenidos de la Educación Física, para así profundizar mas en esta disciplina y a su vez comprender la estrecha relación entre deporte y emoción.

\section{1.- Actividades artísticos expresivas}

Siguiendo a Marín \& De la torre (1991), en su manual para la creatividad, podemos comprender los beneficios de este contenido a las emociones.

\section{Las actividades artísticos expresivas como método para la creatividad}

Realizamos proyectos surgidos desde dentro, siendo una forma de comunicarse en liberta, por placer estético, satisfaciendo así nuestras inclinaciones más intimas. Por medio del arte el sujeto descubre, experimenta, y recrea los objetos, desde su óptica persona. Cuando no es una imitación de una reproducción creada por otra personal, se puede alcanzar niveles muy elevados de creatividad, esta comenzando en la infancia siendo expresiva, y convirtiéndose en productiva e innovadoras en adultos. Logan \& Gracía (1980).

\section{Las actividades artísticos expresivas como expresión de emociones}

Desarrollando la sensibilidad y los sentimientos de forma constructiva, jugando un papel importante en la creatividad. Estas emociones se puede reproducir mediantes 
interpretaciones, movimientos, colores, formas, etc. pero antes de ser representada y expresada por el sujeto se debe interpretar. Potenciar las actividades artísticos expresivas, es impulsar la sensibilidad personal, la percepción diferencial, y el desarrollo de la dimisión afectiva.

\section{Las actividades artístico expresivas como comunicación creativa}

Mediante estás actividades se desarrolla códigos no verbales, mediante los cuales resulta mas fácil trasmitir las emociones, sentimientos, actitudes, y valores a las personas de nuestra lengua.

\section{Las actividades artísticos expresivas, como desarrollo estético}

A través del arte, el individuo aprende a valorar lo creativo, se sensibiliza ante la forma estética, agudiza su percepción visual y logra el aprecio de las formas artísticas.

\section{Las actividades artísticos expresivas como experiencia}

En estas actividades se da un sentido creativo a las experiencias vividas. Se fuerza las relaciones y proporciona significativamente todo lo que le rodea, el sujeto reorganiza mentalmente su entorno, lo interpreta de forma personal y lo comunica.

\section{Las actividades artísticos expresivas como autorrealización}

El individuo busca satisfacer su necesidad de llegar a ser lo que pretende, siendo el arte un camino real de autoexpresión, de transformación de los propios pensamientos y emociones. Maslow (1982).

\section{2.- Actividad física y salud y Condición física y salud}

El ministerio de Sanidad, Servicios Sociales e Igualdad, presenta campañas a nivel educativo para intentar disminuir la epidémica clasificada por muchos del Siglo XXI la Obesidad, Moreno, Monereo \& Álvarez, (2000); Bosch, Alfonso \& Bermejo (2002). A la hora de intentar reducir está plaga, y mejorar nuestro estilo de vida o conjunto de hábitos de una persona, se incide en los modelos teóricos determinante en la práctica de la Actividad Física: como son: los modelos de creencia sobre la salud, Becker \& Maiman (1975), teoría de la autosuficiencia, Bendura (1986), modelo de la actividad Física, Sonstroen (1978), teoría de la acción razonada, Arzen \& Fishbien (1997), modelo trasteórico, Prochaska \& Velicer, (1997), teoría cognitivoSocial, Gottlieb \& Baker (1986), modelo ecológico, Spence \& Lee (2003), intentando 
todos producir un cambio de hábitos en la conducta de los sujetos a partir de la motivación intrínseca.

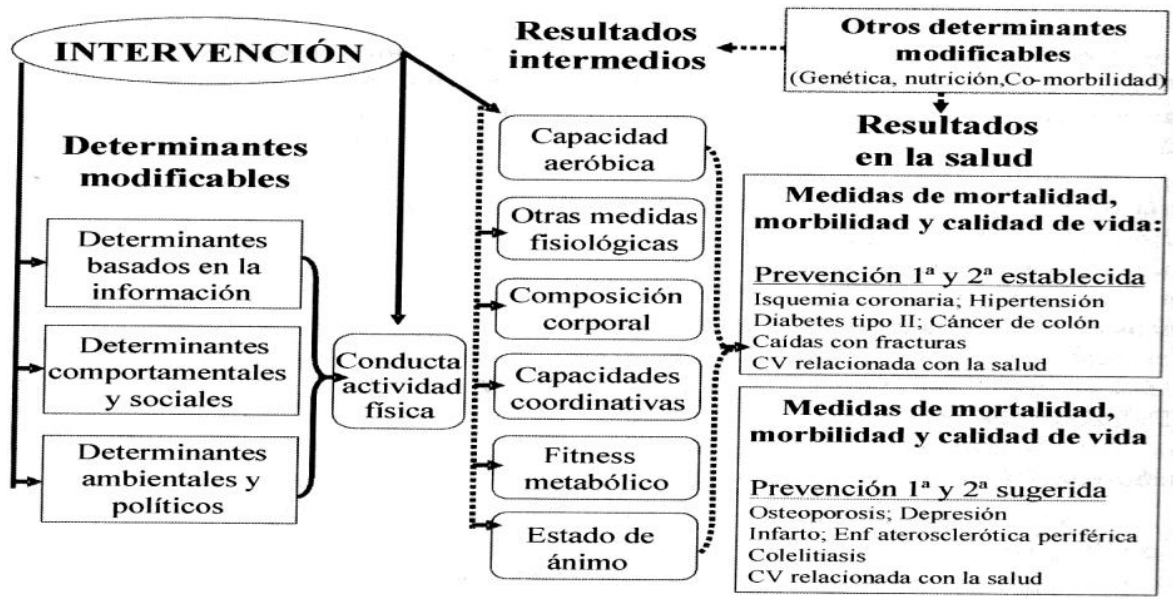

Figura 4. Modelos de intervención que se han mostrado efectivos a la hora de promocionar la actividad física. (Peréz-López 2009) Adaptado de Kahn y cols. (2002).

Estos cambios como se muestra en la figura 4 deben ser tratados desde el comportamiento y los estados de ánimo de los sujetos, ya que por pertenecer a poblaciones especiales como la anteriormente mencionada, obesidad, se pueden sentir reprimidos ante el grupo, sin suficiente energía y alegría por expresar sus sentimientos y en algunos casos hasta marginados.

\section{3.- Juegos y actividades deportivas y Juegos y deportes}

Siguiendo uno de los sietes principios del entrenamiento, principios de las diferencias individuales, podemos inducir las diferencias psico-físico-técnico-táctico, antropométricas, familiares y culturales de cada sujeto, haciendo estas desigualdad competente en cada una de las Inteligencia múltiples y en este caso en cada una de las especialidades deportivas, considerando que todos los individuos no tienen la misma soltura a desarrollar todas las acciones tácticas con las misma eficacia, habrá sujetos mayores dotados que otros, por las diferencias anteriormente mencionadas.

Podemos considerar la inteligencia deportiva como, el conocimiento y control del mayor número de condiciones que determinan el éxito en una acción eficaz, de la modalidad deportiva que estamos disputando, o del juego con el cual nos estemos recreando. Del Pino, Gómez, Moreno, \& Gálvez, (2009).

Durante la época de iniciación deportiva donde muchos patrones básicos todavía no se han consolidados, puede que el bloqueo se haga presente en los sujetos, exponiendo emociones básicas como la ansiedad por fallar en una acción, la 
rabia por no conseguir el objetivo que te plantees a corto o largo plazo y no obtener progresos en esa modalidad deportiva, o la desilusión por no asumir el rol de perdedor durante el juego. Figura 5

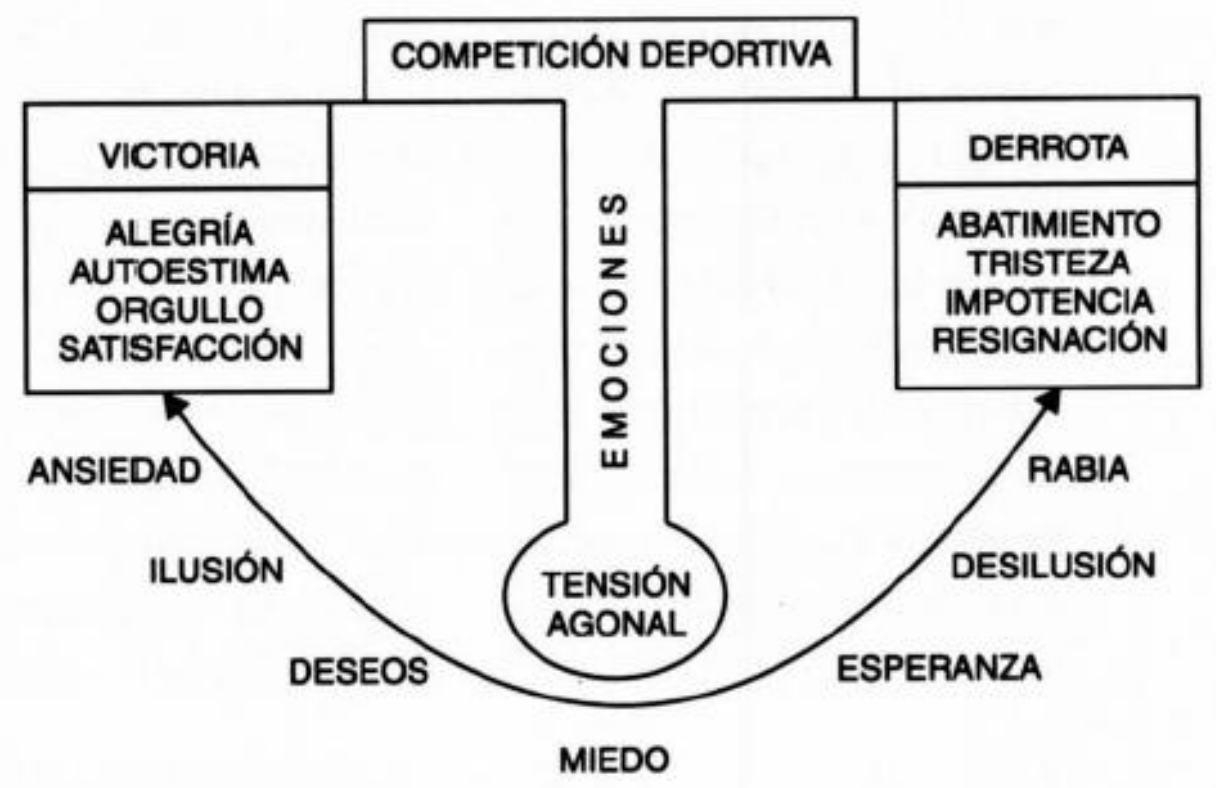

Figura. 5. Estamos emocionales derivado de la competición deportiva. Lagardera (1999).

Estos estados emocionales contradictorios, producen una desmotivación, Gordillo (1992) citado por (Gonzales, Tabernero \& Márquez 2000), en el individuo acentuando el abandono, o la emoción de asco hacia una actividad, es hay donde debemos incidir los docentes, haciendo hincapié en que los sujetos vivencies experiencia positiva, Morilla (1994) siguiendo a (Gonzales, Tabernero \& Márquez 2000), para crear una mayor adherencia a la práctica deportiva.

Estas vivencias agradables hacen florecer en los sujetos emociones positivas que se derivan de estas actividades, tales como la socialización y la amistad en los juegos o deportes de equipos, o el sacrificio y la alegría por conseguir y alcanzar las metas.

Destacar la importancia de los deportes abiertos, "donde prima la creatividad, desde las posibilidades que brinda el pensamiento divergente más que el entrenamiento del automatismo y la respuesta cerrada." Del Pino, Gómez, Moreno \& Gálvez (2009). 
En actividades donde el individuo está totalmente en movimiento y cambiando la posición al igual que sus compañeros, rivales, y entorno la incertidumbre juega un papel fundamental, Jiménez-Sánchez, (2004), siendo importante, el control del estrés, la atención, y la autoconfianza en edades tempranas, Gimeno, Buceta \& Pérez (2007).

En una investigación realizada por Gonzales, Tabernero \& Márquez (2000), indagaron sobre los motivos que llevaron a los niños a realizar dos deportes como el Tenis y el Fútbol. El resultado del estudio muestra que los motivos se acercan mas hacia características intrínsecas tales como aprender, divertirse, estar en forma, hacer nuevas amistades, y mejorar, dejando en un segundo plano lo extrínseco como ganar, ser importante o popular.

Parece difícil concretar características psicológicas especificas de un deportista, pero en un estudio realizado por . Del Pino, Gómez, Moreno, \& Gálvez, (2009), nos marca pinceladas según los resultados obtenidos del perfil psicológico del sujeto, con las sucesivas particularidades:

- Muestran una alta competencia en inteligencia corporal, por encima de cualquier otra.

- Presenta valores elevados de inteligencia interpersonal e intrapersonal

- Las demás inteligencia representa un papel secundario en el perfil de inteligencia múltiples típico para personas deportistas de ambos sexos

- La inteligencia lingüística y musical son las que se obtuvieron menor puntuación

Siguiendo está investigación de . Del Pino, Gómez, Moreno, \& Gálvez, (2009), se puede inducir el subtipo de inteligencia y el perfil del deportista según algunas modalidades deportivas.

En futbolistas, destacan en inteligencia kinestésica e interpersonal, siguiéndole la matemática, visual, naturalista e intrapersonal, y una baja inteligencia lingüística y musical.

Jugadoras de baloncesto, sobresalen en inteligencia corporal e interpersonal seguida de la intrapersonal. Siendo la inteligencia musical en la que menos destacan.

Nadadores, predominan la inteligencia kinestésica e intrapersonal, siguiéndole la matemática, visual musical e interpersonal, obteniendo valores bajos en la lingüística y natural. 
Atletas. Obteniéndose pocas diferencias según la prueba que realicen, y al igual que las modalidades estudiadas se incide en la inteligencia corporal, interpersonal e intrapersonal.

Mencionar que la muestra no representa a la población correspondiente, por lo tanto no se puede extrapolar el perfil que se ha mencionado a todos los practicante de esa modalidad deportiva, pero si nos podemos guiar, para saber, con que tipo de sujetos nos podemos encontrar y las inteligencia que podemos desarrollar.

\section{4.- Expresión corporal}

Se puede considerar la expresión corporal como el conjunto de técnicas que utiliza nuestro cuerpo para relevar nuestro mundo interno, sensaciones, emociones, ideas, etc., ayudando a mejorar nuestro bienestar personal mediante la toma de conciencia y aceptación de nuestro propio cuerpo. Salzer, 1984, \& Satokoe 1984, citado por (Ruano \& Barriopedro 2006).

El objetivo de la expresión corporal es elaborar y crear nuevos caminos para que el alumno no solo disponga de los conocimientos necesarios para el control y manejo corporal, sino también para que pueda desarrollar creativamente su imagen, su propio lenguaje, su capacidad de relación y captación de estímulos, ya sean auditivos, rítmicos, visuales, composición o relaciones espaciales, así como la recepción y trasmisión de los estados emociones y climas colectivos Schinca, 1988:7, citado por (Ruano \& Barriopedro 2006).

Estos caminos que nos llevan a trasmitir las emociones son los contenidos que se trabajan dentro de la asignatura que a continuación expondremos:

\section{El mimo y el juego simbólico}

Surge de la relación con el resto de compañeros y de exteriorizar y compartir nuestras emociones con ellos, esta expresión de las emociones fomenta la creatividad del sujeto.

\section{El Juego dramático}

El alumno se conoce, mejora sus relaciones interpersonales, facilita la expresión de los estados de ánimos, sentimientos, e interacción con el medio que le rodea, Benítez y Hernández (1999). 


\section{Los juegos cantados}

Favorecen la expresión y vivencia de las emociones, mediante la comunicación.

\section{La toma de conciencia y aceptación de nuestro cuerpo}

Nos proporciona un bienestar personal mediante la liberación y control de las emociones (relajación, respiración, etc.), en muchos casos cuando conseguimos una fluidez emocional se produce una desinhibición ante las propuestas de expresión corporal. Canales (2007).

En la aceptación de nuestro cuerpo entra en juego los marcadores somáticos, siendo un cambio postural que refleja el estado de ánimo del sujeto, tanto positivo como negativo, esto puede incidir en las decisiones tomadas en un momento determinado. La respuesta emocional es subjetiva y somática, asociándose a una acción o conjunto de estímulos, que puede influir en una conducta futura. Estos marcadoras son cambio musculares, vegetativos, neuroendronicos y neurofisiológicos, proporcionando señales inocentes, agilizando y facilitando la toma de decisión, antes incluso que el sujeto la pueda explicar (Martínez, Sánchez, Bechara, \& Román, 2006).

\section{La danza}

De carácter socializador e integrador, los alumnos se sienten alegres de poder inventar, crear, y apreciar que son autónomos, a través de la danza. El máximo exponente en este caso es la emoción de nuestro cuerpo sintiéndose totalmente libres para exteriorizar lo que siente.

En una investigación realizada por Canales. I (2007), analizó las emociones que se trasmitía en las sesiones de la asignatura de Expresión Corporal, mediante ejercicios donde predominaban el tacto y la mirada. Esta investigación se realizo con alumnos de la Diplomatura de Educación Física de la Universidad de Zaragoza. Las experiencia se analizaron a partir de los testimonios de alumnos y alumnas.

\section{La mirada}

En la mayoría de los casos condiciona el comportamiento ya que el sujeto observado en muchas ocasiones no exterioriza sus emociones en su totalidad, debido a las siguientes situaciones. 


\section{El sujeto observado se siente enjuiciados por los demás}

Mostrándose inquieto e inseguro por la observación de los restantes ante su actuación.

\section{Ridiculizados por realizar la actividad}

Algunos alumnos restringen su conducta sintiéndose incomodos ante la situación creada, mientras otros tanto se sienten reforzados.

\section{La desconfianza con los compañeros}

Según el vinculo de afinidad que tengas con la personas que realice la acción te proporcionara mas tranquilidad o no.

\section{La sala de expresión corporal}

Los alumnos condicionan las conductas según sean observado desde el exterior, o encontrándose en una sala totalmente cerrada en la cual las únicas personan que pueden percibir sus conductas sean las allí presentes, sintiéndose de esta forma más seguro a exteriorizar sus sentimientos.

\section{Las zonas corporales observadas}

Mostrando nerviosismo cuando son observados a los ojos y mas alivio cuando tiene el rol de observador o la zona elegida son las manos o pies de menor implicación social.

\section{La diacronía en la ejecución}

Dependiendo si realizan los ejercicios todos a la vez o si existe un orden establecido en la ejecución.

\section{Diferencia de comportamiento según los géneros}

Las chicas muestran más soltura en sus actuaciones, en cambio los chicos se sienten más bloqueados y aluden a una falta de fluidez.

\section{La singularidad y el mimetismo de los compañeros}

Diferentes respuesta y exteriorización de las emociones ante un estimulo, en muchas ocasiones los alumnos se imitan unos a otros, eligiendo cada uno diferentes vías de exploración, y ser creativos.

\section{El tacto}




\section{Compromiso dependiendo de la parte del cuerpo que haya que tocar}

Mostrando rubor y bloqueo del alumno por no querer invadir la intimidad del compañero.

\section{Vinculo de afinidad con el sujeto que se realiza la acción}

Lazo de unión existente entre receptor y emisor. La amistad que se tiene con el otro compañero hará sentirse al sujeto seguro o no, sobre todo en ejercicios, con desplazamientos o balanceos... donde existe algo de riesgo.

\section{Prudencia en la manipulación}

Sin intentar dañar al compañero que manipulan, la cautela se pronuncia cuando es el alumno el que realiza la actividad con una compañera.

\section{La presión del contacto}

Decisivo para muchos alumnos para tener una experiencia grata 0 desagradable y dependiendo si la fricción la realiza uno o mas compañeros. Un gran número de alumnos destaca que la concentración en la relajación te ayuda disfrutar de esa presión o no.

\section{La armonía de contacto}

Condicionada por si la ejecución, se realiza de manera lenta o suave, provocando sensaciones diferentes, a mayor lentitud sensación de suavidad y tranquilidad, cuando la actividad se realiza a mayor velocidad el sujeto que percibe la fricción puede ponerse mas nervioso.

\section{La diferencia de género}

Los alumnos comenta que cuando el tacto lo reciben de una compañera le resulta mas gratificante, en cambio cuando se cambia los roles las alumnas lo tildan de superficial y en algunos casos de malestar por no disfrutar al $100 \%$ de la actividad, existiendo cautela a la hora de tocar en ciertas partes del cuerpo, por el temor de ellos a dañar o invadir la intimidad sus compañeras.

Se considera que la expresión corporal, es un canal perfecto para expresar nuestras emociones, ya que une la comunicación y expresión e incluso puede llegar a modificarlas. A partir de este contenido podremos liberar tensiones acumuladas, y expresarlas a través de nuestro cuerpo. 
Los alumnos deben de ser capaces de reconocer lo que siente en cada momento y distinguir si el estado emocional que están viviendo en ese instante, proviene de un pensamiento negativo o positivo.

\section{5.- Actividades en el medio natural}

Tomando como partida la definición Amstrong (1980) citado por (Miranda, Lacasa \& Muro1995), podemos definir este tipos de acciones como actividades que procuran una experiencia humana, con los elementos del medio ambiente posibilitándote la oportunidad de salir de la vida cotidiana y vivir aventuras, siendo libres para expresar las emociones en una sociedad sin emociones Freixa (1995), en pleno contacto con la naturaleza Olivera-Bertrán \& Olivera-Bertrán (1999).

Autores como. Miranda, Lacasa \& Muro, (1995) exponen la necesidad del contacto con la naturaleza en una sociedad demasiada controlada y rutinaria. La vida no puede ser entendida como algo racional, pues deriva en aburrimiento y falta de autenticidad de la vida cotidiana Simmel (1965), citado por Miranda, Lacasa\& Muro, (1995). En esta misma línea sigue Elías \& Dunning (1986) citados por Miranda, Lacasa\& Muro, (1995), exponiendo al ser humano como sumiso del Estado, ente que no facilita las expresión de las emociones.

Siendo una de las olvidadas en la enseñanza por ignorancia o temor del docente, estas actividades promocionan un amplio paquete de fuertes e insólitas emociones y sensaciones totalmente diferentes e imposible de reunir en otros contenidos, Olivera-Bertrán \& Olivera-Bertrán (1999), por la variedad de actividades presentes y la peculiaridad y diferencia existente entre cada una de ellas, Freixa. C (1995), pues podemos realizar actividades en diferentes medios, agua, tierra y aire, ofreciéndonos cada uno diferentes sensaciones, como por ejemplo, la incertidumbre de no saber como te vas a encontrar la mar y el viento en actividades acuáticas, la adrenalina soltada en una actividad como el puenting o la paz interior que te produce una inmersión en el agua al realizar submarinismo.

Características de las actividades en el medio natural según, Miranda, Lacasa \& Muro, (1995):

- No están sujetas a reglamentación fija, lo cual proporciona una sensación de libertad que no te lo da los deportes reglados

- No se rige por horarios. Se puede practicar cuando se deseen 
- Su forma de practica, intensidad, modo o ritmo puede variar a gusto del usuario, lo que hace una práctica mucho mas lúdica proporcionando un estado emocional de tranquilidad

- Son originales, cambiantes y sobre todo creativas

- Han cambiado el tradicional paradigma del esfuerzo, por el paradigma de el equilibrio y dar las gracias

- Busca el placer sensomotor, por lo que el estado emocional del sujeto es de confianza plena hasta llegar a esa respuesta

- La aventura está presente en toda actividad y con ello la ruptura de la vida cotidiana. (Olivera-Bertrán \& Olivera-Bertrán 1999; Freixa, 1995 \& , Miranda, Lacasa \& Muro, 1995)

- La sensación de riesgo por cumplir en nuestras sociedad una función fundamental de reguladores catárticos, de instrumentos para la gestión controlada de las emociones. Freixa, (1995)

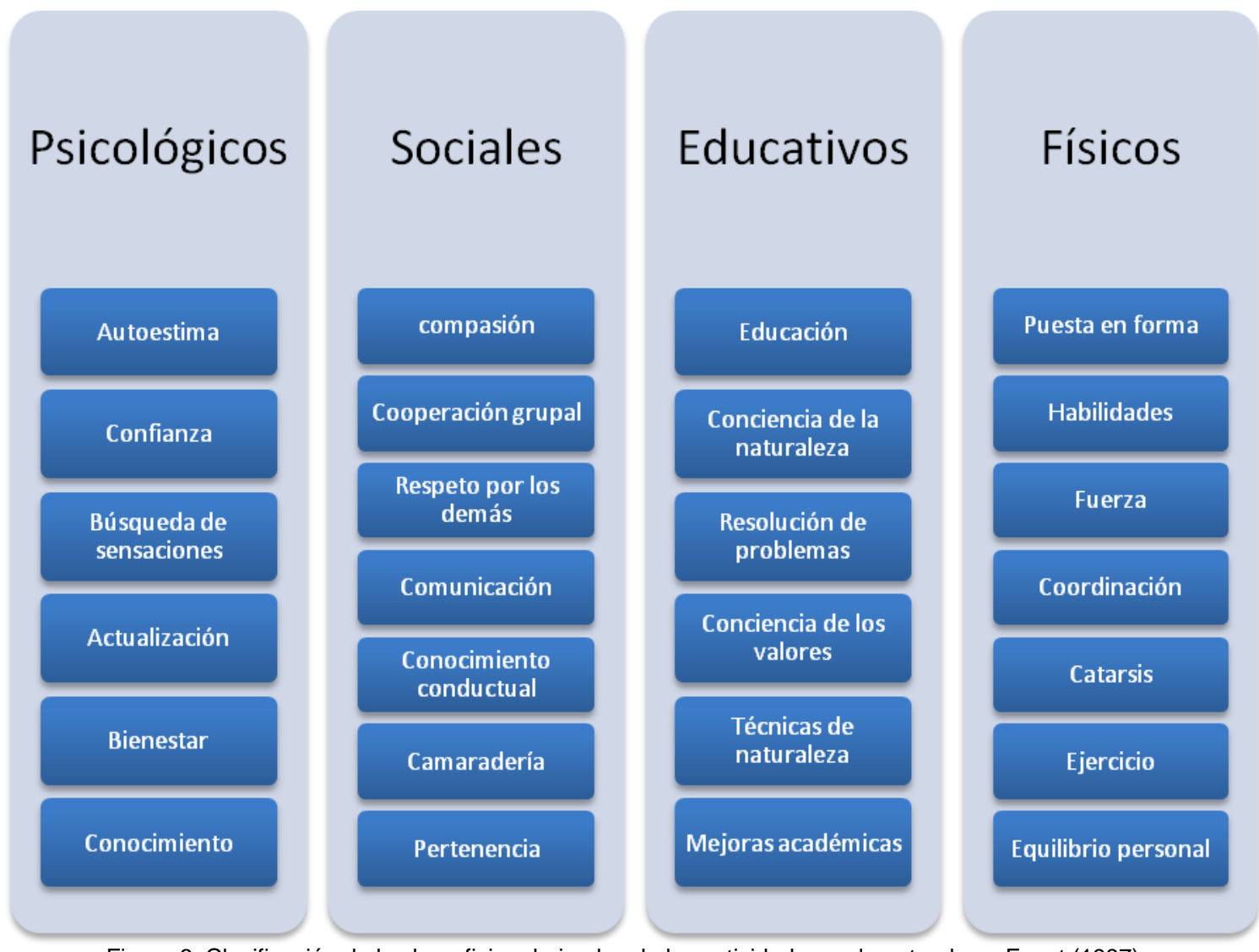

Figura 6. Clasificación de los beneficios derivados de las actividades en la naturaleza. Ewert (1987).

Los beneficios de este tipos de actividades son múltiples como podemos observar en la figura 6 es por este motivo por el cual Freixa. C (1995) las consideras 
actividades miméticas, por que si las conseguirnos extrapolar a nuestra vida cotidiana el beneficio será positivo.

\section{8.- EL ENTRENAMIENTO DE LAS EMOCIONES}

Como hemos comprobado con anterioridad muchas personas encuentra dificultades para superar los retos que se le presenta, muchas veces a nivel físico y otras a nivel psicológico o emocional siendo incapaz de superar el desafío planteado. La respuesta al problema físico muchos profesionales de las ciencias del deporte lo achacarían a la falta de entrenamiento, pero al igual que el competente motriz se puede moldear y entrenar el componente psicológico y emocional de igual manera se puede ejercitar. Para potenciar estas destrezas hay que entrenar las siguientes habilidades, Carrascosa (2003):

- El control de la atención y concentración.

- El ajuste de la tensión y activación neurofisiológica.

- Afianzar la autoconfianza, sintiéndose seguro de uno mismo y de sus cualidades.

- Autocontrol emocional, controlando aquellas emociones que nos impida rendir al máximo de nuestras posibilidades, encaminándonos al fracaso y hacia la incapacidad de un rendimiento optimo de nuestra acción.

- Actitud, ideas y valores relacionados con la actividad desarrollada.

Este tipo de entrenamiento debe ser exigente a nivel emocional y adecuarse a las demandas en la actividad, de esta forma seremos capaces de preparar psicológica y emocionalmente a nuestro sujeto para el esfuerzo requerido. No solo debemos estar alerta en nuestras horas de entrenamiento a nivel emocional, pues nuestra vida cotidiana puede ser un buen campo de actuación para preparar nuestras emociones a la práctica que hemos de realizar.

\section{9.- CONCLUSIONES}

Respondiendo a los objetivos planteando al principio del trabajo, ha quedado patente la utilidad de la inteligencia múltiple corporal-kinstésica, en los contenidos de Educación Física y su trabajo con las emociones e inteligencia emocional en el ámbito educativo. 
Como docentes debemos potenciar las emociones que incite cada contenido, pues cada uno tiene unas características diferentes para trabajar en el. Un mal estado emocional de los sujeto puede incidir en una realización técnica o táctica ineficaz provocando experiencias negativas de los alumnos.

Las emociones o habilidades que el sujeto exprese o desarrolle en las clases de Educación Física, debemos de ser capaces de hacer que lo extrapole ha su vida cotidiana, para una mayor realización de esta, siendo las clases de Educación Física, un medio ideal para, sentir y expresar de todo tipos de estados de ánimos.

\section{0.- BIBLIOGRAFÍA}

Bosch, X. Alfonso, F. \& Bermejo, J. (2002). Diabetes y enfermedad cardiovascular. Una mirada hacia la nueva epidemia del siglo XXI. Revista Española de Cardiología, 55 (5), 525-527.

Carrascosa, J. (2003). Saber Competir. Claves para soportar y superar la presión. Madrid; Gymnos.

Del Pino, Medina, J.M. Gómez, Milán, E. Moreno, Ríos, S \& Gálvez, García, G. (2009). Inteligencia múltiples y deporte. Revista Apunts, 95, 5-13.

Freixa, C. (1995). La aventura imaginaria. Una visión antropológica de las actividades de aventura en la naturaleza. Revista Apunts, 41, 36-43.

Gardner, H. (1993). La estructura de la mente. Teoría de las múltiples Inteligencia. México: fondo de Cultura Económica.

Gimeno, F. Buceta, J.M \& Pérez-Llantada, M.C. (2007). Influencia de las variables psicológicas en el deporte de competición: evaluación mediante el cuestionario. Características psicológicas relacionadas con el rendimiento deportivo. Revista Psicothema, 19 (4), 667-672.

González, G. Tabernero, B \& Márquez, S. (2000). Análisis de los motivos para participar en fútbol y en tenis en la iniciación deportiva. Revista Motricidad, 6, 4766.

Jiménez, Sánchez, A. C. (2009). La táctica individual o el problema sobre qué y cómo deciden los deportistas en los deportes colectivo. Seminario internacional de táctica y técnica deportiva: la táctica deportiva y la toma de decisiones.

Lagardera, Otero, F. (1999). La lógica deportiva y las emociones. Sus implicaciones en la enseñanza. Revista Apunts, 56, 99-106. 
Levav, M. (2005). Neuropsicología de la emoción. Particularidades en la infancia. Revista Argentina de Neuropsicología. 5. 15-24.

Marín, R. \& De la Torre, S. (1991). Manual de la Creatividad. Barcelona: Vicens Vives.

Martínez, Selva, M. Sánchez, Navarro, J.P. Bechara. A. \& Román, F. (2006). Mecanismos cerebrales en la toma de decisión. Revista de Neurociencia. 42 (7), 411-418.

Miranda, J. Lacasa, E \& Muro, I. (1995). Actividades físicas en la naturaleza: un objetivo a investigar. Dimensiones científicas. Revista Apunts. 41, 53-69.

Moreno, Esteban, B. Monereo, Megías, D \& Álvarez, Hernández, S (2003) La epidemia del siglo XXI. 2ª Edición. Revista Nutrición Hospitalaria. 1, 51-52.

Olivera, Bertrán, A \& Olivera, Bertrán, J. (1999). Las actividades físicas de aventura en la naturaleza. Estudio de la oferta y la demanda en el sector empresarial. Revista Apunts, 57, 86-94.

Pérez, López, I.J. (2009). Salud. Educación Física y Juegos del Rol. Sevilla: Wanceulen.

Ruano, Arriagada, M.T \& Barriopedro, Moro, M. I (2004). Impacto de la expresión corporal sobre las emociones en el ámbito educativo. Monográficos del III Congreso de la Asociación. Volumen 14. Valencia.

Rueda, B. (2011). Máster En Educación Físico Deportiva. Apuntes de la asignatura: Intervención en la enseñanza de la expresión corporal. Universidad de Huelva. Inédito. 